\section{Befundung genetischer Tests}

\section{J. Arnemann}

Abteilung Molekulargenetik, Labor Dr. Wisplinghoff, Köln, Deutschland

\section{Synonym(e) Analyse-Ergebnis}

Englischer Begriff reporting results of genetic testing

Definition Die Befunde genetischer Tests sind formelle medizinische Dokumente vom analysierenden Labor an den beauftragenden Arzt oder die beauftragende Klinik und sollten strukturell und inhaltlich den internationalen Leitlinien folgen.

Beschreibung Im Vordergrund eines jeden genetischen Befundes sollte eine eindeutige, präzise und korrekte Antwort auf eine klinische Frage samt zugehöriger Interpretation stehen.

Die Qualität und die Richtigkeit des Befundes sind aber auch wesentlich vom einsendenden Arzt bzw. der präzisen Untersuchungsanforderung abhängig. So muss der Auftrag für das genetische Labor genau formuliert werden und die klinische Differenzialdiagnose und gegebenenfalls weitere klinische Befunde enthalten.

Der Befund sollte einem im Labor vorgegebenen formellen Rahmen folgen und administrative Informationen beinhalten. Hierzu gehören Name (Logo) und Adresse des analysierenden Labors, Datum des Probeneingangs und/oder Probenabnahme und des Befundausgangs, Unterschriften und Ausbildungs- bzw. Weiterbildungsstatus von mindestens 2 autorisierten Mitarbeitern sowie Name und Anschrift des Einsenders, Untersuchungsanforderung, Name, Geschlecht,
Geburtsdatum, Labor-Identifikationsnummer, Untersuchungsmaterial und, wenn möglich, auch ethnische Zugehörigkeit des Patienten.

Der individuelle und auf die diagnostische Anforderung ausgerichtete Befundteil sollte den Grund für die Überweisung zur Analyse nochmals aufgreifen und die Spezifikation und Sensitivität des hierfür eingesetzten Tests beschreiben. Kommerziell erhältliche Testkits müssen ebenso erwähnt werden, wie die getesteten Teilabschnitte eines Gens (z. B. Exons) und die für die Auswertung hinterlegte offizielle Referenzsequenz des Gens in der Gendatenbank, wie beispielsweise EMBL oder Genbank.

Im Mittelpunkt steht jedoch das eigentliche Ergebnis der Testung, das kurz, präzise und unzweifelhaft formuliert sein soll und die gefundenen Sequenzabweichungen gegenüber der Referenzsequenz mit der von der HGVS, der Human Genome Variation Society (http://varnomen.hgvs.org), vorgegebenen Nomenklatur beschreibt.

Dem Ergebnis muss eine detaillierte Interpretation folgen, die aus den genetischen und klinischen Daten eine Beurteilung hinsichtlich einer möglichen Pathogenität der gefundenen Sequenzvarianten zulässt.

Zusammenfassend muss der Befund zu einem genetischen Test ein klares Ergebnis mit einer sicheren Interpretation vor dem klinischen Hintergrund des Patienten berichten und sollte nach einer Faustregel nicht den Umfang einer Seite überschreiten.

\section{Literatur}

Claustres et al (2014) Recommendations for reporting results of diagnostic genetic testing (biochemical, cytogenetic and molecular genetic). Eur J Hum Genet 22:160-170 